\title{
An Overview on Quantum Cascade Lasers: Origins and Development
}

\author{
Raúl Pecharromán-Gallego \\ Additional information is available at the end of the chapter
}

http://dx.doi.org/10.5772/65003

\begin{abstract}
This chapter presents an introductory review on quantum cascade lasers (QCLs). An overview is prefaced, including a brief description of their beginnings and operating basics. Materials used, as well as growth methods, are also described. The possibility of developing GaN-based QCLs is also shown. Summarizing, the applications of these structures cover a broad range, including spectroscopy, free-space communication, as well as applications to near-space radar and chemical/biological detection. Furthermore, a number of state-of-the-art applications are described in different fields, and finally a brief assessment of the possibilities of volume production and the overall state of the art in QCLs research are elaborated.
\end{abstract}

Keywords: quantum cascade lasers, review, history, operation, fundamentals, materials, photoacoustic spectroscopy, sensors, trace-gas detection, plasma species, cavity ring-down spectroscopy

\section{Introduction}

Quantum cascade lasers (QCLs) are based on a fundamentally different principle to 'classic' semiconductor lasers, that is, they use only one type of charge carriers, electrons, using intersubband transitions, so they can be called unipolar lasers. QCLs were conceived in the early 1970s. First, Esaki and Tsu [1] fabricated the first one-dimensional periodic potential multilayer by periodically varying the composition during epitaxial growth (superlattice). Later, QCLs were proposed by Kazarinov and Suris [2] and finally first demonstrated at Bell Laboratories in 1994 by Faist et al. [3]. Using superlattices leads to both quantum confinement and tunnelling phenomena, the basic processes in QCLs operation. 
A conventional laser diode generates light by a single photon that is generated from an electron interband transition; this means that a high-energy electron in the conduction band recombines with a hole in the valence band, being the energy of the photon determined by the band-gap energy of the material system used. However, QCLs do not use bulk semiconductor materials in their optically active region, but a periodic series of thin layers of varying material composition forming a superlattice, which leads to an electric potential that changes across the length of the device (one-dimensional multiple quantum well confinement), splitting the bandpermitted energies into a number of discrete electronic subbands, making electrons cascade down a series of identical energy steps built into the material during crystal growth, and emitting a photon at every step, unlike diode lasers, which emit only one photon over the equivalent cycle. With an appropriate design of the thickness of these layers, population inversion is achieved between discrete conduction band-excited states in the coupled quantum wells by the control of tunnelling, making laser emission possible. Therefore, the position of energy levels is mainly determined by the thickness of the layers, rather than the material, and thus allowing tuning the emission wavelength of QCLs over a wide range in the same material system. Thus, one electron emits a photon during every intersubband transition within the quantum well $(\mathrm{QW})$ in the superlattice, and then can tunnel into the next period of the structure where another electron can be emitted, leading QCLs to outperform diode lasers operating at the same wavelength by a factor even greater than 1000 in terms of power.

Classically, a QCL is made of a periodic repetition of active sections, which consist of tunnelcoupled quantum wells and injector, where a miniband is formed. As Figure 1 shows [4], from the injector miniband the electrons are injected into the upper laser energy level (4) of the active section, where the laser transition takes place. Afterwards, the lower laser energy level (3) is emptied by longitudinal optical emissions (LO emissions) and the electrons enter the next step by tunnelling.

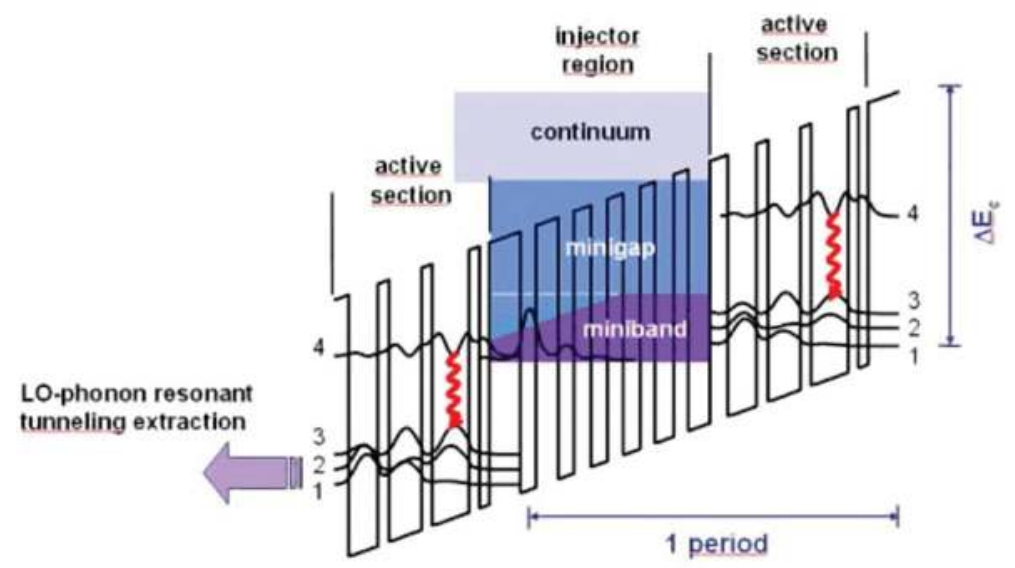

Figure 1. Typical conduction band structure of a QCL [4]. 
Combining materials in the active region, QCLs could be designed to emit at any wavelength over a wide range of the spectrum [5], as the emission wavelength is determined by quantum confinement. Figure 2 shows a typical QCL in operation and some commercial examples. These structures are typically grown using either molecular beam epitaxy (MBE) or metal-organic chemical vapour deposition (MOCVD), being the most used growth mechanisms utilized to grow the alternated different semiconductor layers required for heterostructures fabrication on to a substrate. Ever since the first QCL was fabricated using InGaAs/InAlAs grown over InP substrate [1], other materials have been used in order to fabricate QCL structures, such as GaAs/AlGaAs, InGaAs/AlAsSb, InAs/AlSb, Si/SiGe and GaN-based materials, such as AlGaN/ GaN and AlN/GaN.

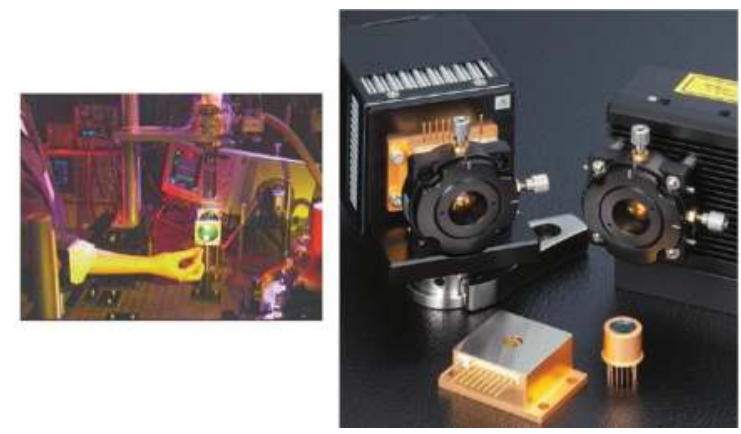

Figure 2. Left: the invisible beam from a high-power quantum cascade laser lights a match in its path. The laser is 2.25 $\mathrm{mm}$ long and $17 \mu \mathrm{m}$ wide, being placed in a cryostat at liquid-nitrogen temperature and emits an optical power in excess of $200 \mathrm{~mW}$ from each facet at a wavelength of $8.0 \mu \mathrm{m}$. Similar devices emit up to $600 \mathrm{~mW}$ at room temperature in pulsed mode. Right: front: mid-IR lasers $(4-10 \mu \mathrm{m})$ for trace-gas analysis and IR molecular spectroscopy in the front; back: set-up examples.

First commercialized a decade later of their first demonstration [6], the key features of these lasers reside in the fact of their high optical power output and, on the other hand, their tuning range and room-temperature operation. Spectroscopy applications are related to gas detection and analysers (pollutants, components, etc.). Other practical uses include industrial control, plasma chemistry and detection, such as collision avoidance radar or poor visibility-driving condition aids. Finally, the 3 to $5 \mu \mathrm{m}$ atmospheric window would make QCLs perfect candidates for substitution of optical fibre in high-speed and free-space communications.

\section{Fundamentals and operating principles}

The main particularity in QCLs is the fact that instead of using bulk semiconductor materials in their optically active region, a periodic series of thin layers are used, that is, superlattices, consisting of a number of quantum well-barrier system equally spaced, introducing a multiple quantum well (MQW) leading to one-dimensional confinement allowing an electric potential variation (band splitting) that results in a number of discrete electronic subbands. In order to achieve the population inversion required for laser emission, it is necessary for a 
proper thickness and composition layer design. These confined energy levels depend on the layer thickness, so the tunability of the emission within the same material relies, in principle, on thickness variation, although multiwavelength QCLs emit by means of different materials within the same structure and multiple resonators [7, 8].

In classical semiconductor laser diodes, electrons and holes recombine across the band gap, thus, generating one photon per $\mathrm{e}^{-}-\mathrm{h}^{+}$pair recombined. However, in QCLs this is not the case, as an electron in the conduction band within a QW emits one photon whenever it undergoes an intersubband transition, that is, thermalization into lower-energy levels, emitting one photon. This electron could tunnel into the next period of the structure, where the mentioned transition happens again emitting another photon. This phenomenon of a single electron emitting multiple photons as it passes through different periods of the structure is called cascade, making the quantum efficiency of QCLs greater than unity and leading to their high optical output power.

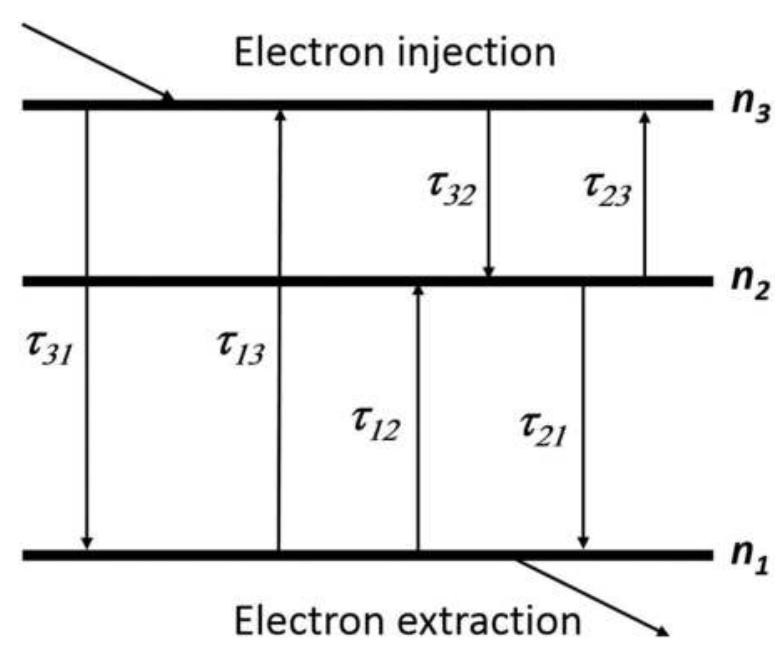

Figure 3. Three-level intersubband transition and scatterings considered in most QCLs.

QCLs are usually three-level lasers, whose level transitions are depicted in Figure 3. In these lasers, the wave function formation is faster than scattering between states; hence, the timeindependent solutions of the Schrödinger equation can be applied, so the system can be modelled using rate equations. Each subband, $i$, is considered to have $n_{i}$ electrons, and a scatter between the initial and the final subband, $f$, will have a scattering rate, $W_{i f}$, and lifetime, $\tau_{i f}$. If no other subbands are populated, the rate equations are described as extraction and injection of electrons, respectively (depicted in Figure 3), whose values are both equal in steady-state conditions, where time derivatives are zero. Hence, the general rate equation for electrons in a subband $i$ within an $N$ level system is where $I=I_{\mathrm{ee}}=I_{\mathrm{ie}}$. At low temperature, absorption is near zero: hence, $\tau_{32}>\tau_{21}$, so $W_{21}>W_{32}$, and $n_{3}>n_{2}$ leading to the existence of population inversion. 


$$
\begin{gathered}
d n_{1} / \mathrm{dt}=n_{2} / \tau_{21}+n_{3} / \tau_{31}-n_{1} / \tau_{13}-n_{1} / \tau_{12}-I_{\mathrm{ee}} \\
d n_{2} / \mathrm{dt}=n_{3} / \tau_{32}+n_{1} / \tau_{12}-n_{2} / \tau_{21}-n_{1} / \tau_{23} \\
d n_{3} / \mathrm{dt}=I_{\mathrm{ie}}+n_{1} / \tau_{13}+n_{2} / \tau_{23}-n_{3} / \tau_{31}-n_{3} / \tau_{23}
\end{gathered}
$$

where $I_{\mathrm{ee}}$ and $I_{\mathrm{ie}}$ are the extraction and injection of electrons, respectively (depicted in Figure 3), whose values are both equal in steady-state conditions, where time derivatives are zero. Hence, the general rate equation for electrons in a subband $i$ within an $N$ level system is

$$
d n_{i} / \mathrm{dt}=S_{f=1}^{N} n_{j} / \tau_{f i}-n_{i} S_{f=1}^{N} \tau_{i f}^{-1}+\mathrm{I}\left(d_{i 0}-d_{i N}\right)
$$

where $I=I_{\mathrm{ee}}=I_{\mathrm{ie}}$. At low temperature, absorption is near zero:

$$
n_{3} / \tau_{32}=n_{2} / \tau_{21}
$$

hence, $\tau_{32}>\tau_{21}$, so $W_{21}>W_{32}$, and $n_{3}>n_{2}$ leading to the existence of population inversion.

The scattering rate between two subbands strongly depends upon the overlap of the wave functions and energy spacing between the subbands. In order to decrease $W_{32}$, the overlap of the upper and lower laser levels is reduced. This is often achieved through designing the layer thickness such that the upper laser level is mostly localized in the left part of the well of the 3QWs active region, while the lower laser level wave function is made to mostly reside in the central and right part of the wells, leading to the so-called diagonal transition. A vertical transition is one which the upper laser level is rather localized in the central and right part of the wells, increasing the overlap and, therefore, $W_{32}$, reducing the population inversion but increasing the strength of the radiative transition and hence the gain. On the other hand, in order to increase $W_{21}$, the lower laser level and the ground level wave functions are designed to obtain a good overlap, and the energy spacing between the subbands is designed such that it is equal to the longitudinal optical (LO) phonon energy so that the resonant LO phononelectron scattering can depopulate the lower laser level. For instance, the LO-phonon energy is around $36 \mathrm{meV}$ in GaAs (which is comparable to the room temperature $k_{B} T$ value of around $26 \mathrm{meV}$ ) and around $91 \mathrm{meV}$ in $\mathrm{GaN}$ [9].

Tunable semiconductor lasers can be produced by using multiple resonators or multisection injection devices [10-12]. In fact, tunable QCLs have been demonstrated [7] some time ago. Moreover, a QCL with a heterogeneous cascade containing two substacks previously optimized to emit at 5.2 and $8.0 \mu \mathrm{m}$, respectively, was presented by Gmachl et al. [13]. On the other hand, single-mode tunable QC distributed feedback lasers emitting between 4.6 and $4.7 \mu \mathrm{m}$ 
wavelength have been reported [14]. These lasers were pulsed, continuously tunable singlemode emission and were achieved from 90 to $300 \mathrm{~K}$ with a tuning range of $65 \mathrm{~nm}$ and a peak output power of approximately $100 \mathrm{~mW}$ at room temperature-so the lasers described by Pellandini et al. [12], Gmachl et al. [13] and Köhler et al. [14] were laser sources for the midinfrared region. In order to realize near-infrared QCLs, optical non-linearity in intersubband lasers has been used to design such lasers emitting at $4.76 \mu \mathrm{m}$, with third harmonic and second harmonic generation at 1.59 and $2.38 \mu \mathrm{m}$, respectively [15].

\section{Materials used and growth methods}

The first QCL in 1994 used InAlAs as the cladding layers due to its low refractive index of around 3.20. The core region, which includes active and injector regions, usually has 500 stacking layers consisting of alternative InGaAs and InAlAs layers with total thickness about 1.5-2.5 $\mu \mathrm{m}$. The average refractive index in this region can be calculated according to the volume fraction of these two constituent materials and is often around 3.35, which is clearly higher than the cladding layers [16]. The confinement factor with typical $\mathrm{Np}$ of approximately 30 is usually around 0.5 . To reduce the optical loss, the cladding layers are usually doped with a low concentration of $5 \times 10^{16} \mathrm{~cm}^{-3}$ and the separate confinement heterostructures (SCHs) are implemented with InGaAs of high refractive index to increase the optical confinement factors. In later designs, the InAlAs cladding layers were replaced by InP because it has a lower refractive index of around 3.10 and a higher thermal conductivity for better heat treatment, which is a critical step for the device performance [16].

So far, the emission wavelength of QCLs has been extended from the near infrared (around $100 \mathrm{THz}$ ) to terahertz regimes. While the longest demonstrated wavelength is $1.6-1.8 \mathrm{THz}$ with $\mathrm{GaAs} / \mathrm{Al}_{0.1} \mathrm{Ga}_{0.9} \mathrm{As}$ system at $80 \mathrm{~K}$ under continuous-wave $(\mathrm{CW})$ operation [17], the shortest wavelength has been extended to $3 \mu \mathrm{m}$ with $\mathrm{In}_{0.53} \mathrm{Ga}_{0.47} \mathrm{As} / \mathrm{AlAs}_{0.56} \mathrm{Sb}_{0.44}$ system at $300 \mathrm{~K}$ under pulsed operation [18]. The highest output power of short-wavelength QCL $(4.6 \mu \mathrm{m})$ under CW operation at room temperature has been demonstrated $(100 \mathrm{~mW}$ and maximum temperature $105^{\circ} \mathrm{C}$ ). All of these state-of-the-art QCLs have been grown by MBE so far, but for industry MOCVD is preferable for mass production. As a result, attempts have been made to grow QCLs by MOCVD. Until now, there are only three groups successfully reporting the growth of QCLs by MOCVD: an InP-based QCL $(\lambda \approx 8.5 \mu \mathrm{m})$ operating in pulsed mode at room temperature, with low-temperature threshold current density in the region of $1500 \mathrm{~A} / \mathrm{cm}^{2}$ [19], an $\mathrm{In}_{0.53} \mathrm{Ga}_{0.47} \mathrm{As} / \mathrm{In}_{52} \mathrm{Al}_{0.48} \mathrm{As}$ QCL $(\lambda \approx 8.5)$ operated in continuous-wave operation at room temperature with an output power of $5.3 \mathrm{~mW}$ [20], and Diehl et al. [21] managed to grow a QCL working in continuous-wave mode above $370 \mathrm{~K}$, with an optical output power of $312 \mathrm{~mW}$ at room temperature and an emission wavelength of $5.29 \mathrm{~mm}$. More effort is still required for MOCVD growers to develop more advanced techniques to compete with MBE. Having said that, room-temperature operation in InAs/AlSb QCLs has been achieved at $4.5 \mu \mathrm{m}$ [22]; more recently, MBE-grown InAs/AlSb on n-InAs (100) substrate QCL operating at $8.9 \mu \mathrm{m}$ has been reported, with a maximum operating temperature of $305 \mathrm{~K}$ [23]. 
QCLs provide one possible method of realizing high-efficiency light emitters in indirect band gap materials such as silicon. Electroluminescence at terahertz frequencies from $\mathrm{Si} / \mathrm{SiGe}$ intrasubband transitions has been demonstrated [24], and recently silicide low-loss (down to $2 \mathrm{~cm}^{-1}$ ) waveguides were designed $[25,26]$. Figure 4 shows a transmission electron microscopy (TEM) image from a Si/SiGe QCL.

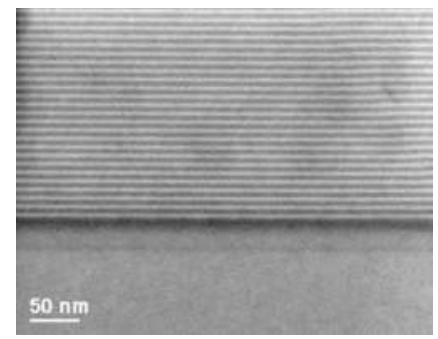

Figure 4. TEM image from a Si/SiGe quantum cascade structure, consisting of 600 periods of $6.5 \mathrm{~nm} \mathrm{Si}{ }_{0.7} \mathrm{Ge}_{0.3}$ quantum wells with 2-nm strained-Si barriers, all grown on top of a $\mathrm{Si}_{0.8} \mathrm{Ge}_{0.2}$ virtual substrate. The quantum cascade laser has a total thickness of $5 \mathrm{~mm}$.

Although GaN-based materials have not been employed to fabricate QCLs, they are also promising materials to be used as such devices. III-V nitrides are known in their wurtzite structure to possess a large spontaneous polarization and piezoelectric constants. As a result, two-dimensional (2D) charges build up at nitride heterointerfaces where the polarization discontinuities occur, causing strong built-in electric fields [27]. Furthermore, GaN is a material with large LO-phonon energy, leading to a thermal population reduction of the lower laser state, a feature desirable for high-temperature operation of terahertz QCLs, as proposed by Diehl et al. [21]. On the other hand, ultrafast LO-phonon scattering in GAN/AlGaN QWs can be useful in order to rapidly depopulate the lower laser state [28, 29]. Lastly, the large LOphonon energy can also increase the lifetime of the upper laser state by reducing the relaxation of electrons with higher in-plane kinetic energy via emission of a LO phonon. Using lowpressure MOCVD, GaN/AlGaN active regions for QCLs have been grown by Huang et al. [30]. GaN/AlGaN active layers are depicted in Figure 5.

Finally, typical phonon frequency redshift is a key indicator of good periodicity of MOVPEgrown GaN/AlGaN QCL structures, $822 \mathrm{~cm}^{-1}$ in the superlattice has been measured, indicating a redshift with respect to the single AlGaN layer [31].

AlN/GaN compound is another possible material to be used to fabricate quantum cascade structures. A suitable method of fabrication is hot-wall epitaxy (HWE) [32], a low-cost, convenient and scalable technique, where the epitaxial layers are grown under conditions as near as possible to thermodynamic equilibrium, allowing a minimum material loss. Inoue et al. [33] grew short-period superlattices consisting of five periods of GaN wells of 10 (or nine) molecular layers (MLs) each with 1 ML AlN barriers, which was designed to emit photons at a wavelength within the mid-infrared range (around $5 \mu \mathrm{m}$ ) [34, 35]. 

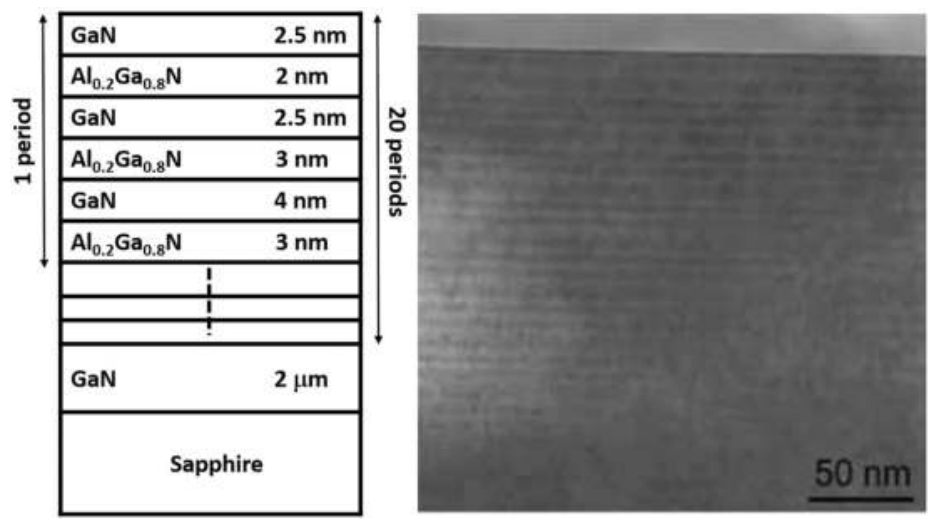

Figure 5. Schematic diagram of the AlGaN QCL active layer structure (left) and cross-sectional TEM (right) of the 20period GaN/AlGaN MQW MQL structure.

\section{Applications}

We could say that QCLs, in short, are eligible to those applications where a powerful and reliable mid-infrared source is required. For instance, most chemical compounds have their fundamental modes in the mid-infrared region $(3-15 \mu \mathrm{m})$ of the electromagnetic spectrum, thus making this range of paramount importance for gas sensing and spectroscopy applications. The so-called two 'atmospheric windows' are two windows corresponding to the ranges 3-5 and 8-12 $\mu \mathrm{m}$, at which the atmosphere happens to show a high transparency leading to remote sensing and detection in those windows. In this section, a brief description of the current applications of QCLs is elaborated, being summarized as subsequently.

\subsection{Trace-gas detection by optical methods in the mid-infrared}

Most trace gases of importance, from products of fossil fuel burning to constituents of human breath, have telltale absorption features in this wavelength range, that is, their 'fingerprint' region of the spectrum, as a result of molecular rotational-vibrational transitions [16]. Narrowlinewidth, tunable semiconductor lasers in this wavelength range are used to spectrally map out and qualitatively and quantitatively detect these trace gases, by a measurement technique called tunable infrared laser diode absorption spectroscopy (TILDAS) [36]. A schematic representation of a TILDAS is shown in Figure 6. The advantage of TILDAS is its high sensitivity and specificity, usually combined with the advantages of the solid-state device approach.

This technique has been successfully introduced in distributed feedback quantum cascade laser (DFB-QCL) structures [37]. DFB-QCLs were first introduced in 1997 [38], providing continuously tunable single-mode laser output, and were demonstrated for the first time in 
trace-gas sensing applications 1 year later [37]. DFB-QCLs operate as follows: a grating with period $L$ is incorporated into the wavewide, lowering the threshold gain (by reducing the outcoming loss) for a different wavelength close to the Bragg wavelength:

$$
\lambda=2 \cdot n_{\mathrm{eff}}(T) \cdot L
$$

where $n_{\text {eff }}$ is the effective refractive index of the waveguide mode, and is a function of temperature. As temperature increases, wavelength shifts to longer values. Changing the heat-sink temperature could control laser temperature; however, the process is slow due to the fact that it implies adiabatically temperature change of a large volume. Tuning rates increase with heatsink temperature current from 0.3 to $0.4 \mathrm{~nm} \mathrm{~K}^{-1}$, for a laser emitting at approximately $5.2 \mu \mathrm{m}$, and from 0.4 to $0.65 \mathrm{~nm} \mathrm{~K}^{-1}$, for a laser whose emitting wavelength is around $8 \mu \mathrm{m} \mathrm{[16].}$

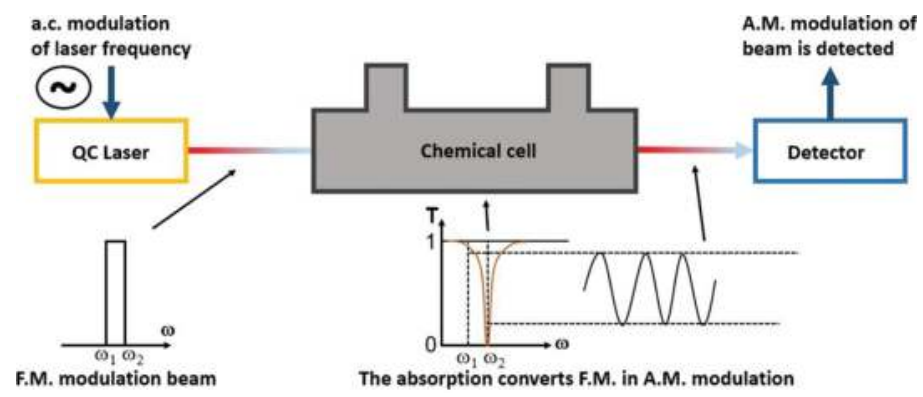

Figure 6. Depiction of a tunable infrared laser diode absorption spectroscopy (TILDAS).

\subsection{Absorption measurements}

In direct absorption measurements, the change in intensity of a beam is recorded as the latter crosses a sampling cell where the chemical to be detected is contained, making this measurement technique quite simple. In a version of this technique, the light interacts with the substance through the evanescent field of a waveguide or an optical fibre.

Namjou et al. [37] used the first DFB-QCL for gas detection purposes. The laser was operated at room-temperature conditions in order to measure mid-infrared ( 1 around $7.8 \mu \mathrm{m}$ ) absorption spectra of gases $\mathrm{N}_{2} \mathrm{O}$ and $\mathrm{CH}_{4}$, diluted in $\mathrm{N}_{2}$ and prepared in a 10-cm long single pass gas cell, using a wavelength-modulation technique. The noise equivalent sensitivity limit of the measurement was $50 \mathrm{ppm}$. Sharpe et al. [39] used a DFB-QCL emitting at 5.2 and $8.5 \mu \mathrm{m}$ to carry out direct absorption measurements of $\mathrm{NO}$ and $\mathrm{NH}_{3}$, respectively. Williams et al. [40] measured the intrinsic linewidth of several DFB-QCLs emitting at around $8 \mu \mathrm{m}$, by observing fluctuation of the collected optical intensity when the laser beam was passing through a sample cell containing $\mathrm{N}_{2} \mathrm{O}$, with a well-known absorption features as the laser was being tuned to the side of one such absorption line. Finally, Kosterev et al. [41] developed a variable-duty cycle 
and quasi-CW frequency-scanning technique for DFB-QCLs, which relieves many of the thermally induced effects of pulsed operation. Combining this laser with a 100-m multipass gas cell and zero-air background subtraction, a detection sensitivity close to 1 ppb concentration levels was achieved; these authors also demonstrated, by using QC-DFB lasers, highsensitivity detection of simple molecules when a spectral resolution of approximately $300 \mathrm{MHz}$ is sufficient [42].

\subsection{Cavity ring-down spectroscopy}

Cavity ring-down spectroscopy (CRDS) is used to measure the concentration of some lightabsorbing substance, typically a gas. A short pulse of laser light is injected into the cavity, bouncing back and forth between the mirrors facing each other that make up the cavity. Typically $0.1 \%$ of the laser light comes out of the cavity and can be measured whenever the light hits one of the mirrors. Hence, as some light is lost on each reflection, the amount of light hitting the mirror decreases each time, leaking a percentage through. An absorbent medium is placed in the cavity, making light undergo fewer detections before it is extinguished. In short, CRDS measures the time it takes for the light to drop for a certain percentage of its original amount, and this ring-down time can be converted to a concentration, having two main advantages: fluctuations in the laser do not affect the measurement (the ring-down time does not depend upon the brightness of the laser), and due to its long pathlength, it is very sensitive, since the light reflects many times between the mirrors.

Paldus et al. [43] reported the use of a $126 \mathrm{~mW}$ CW operated DFB-QCL emitting at around 8.5 $\mu \mathrm{m}$ for CRDS of ammonia diluted in nitrogen. A sensitivity of $3.4 \times 10^{-9} \mathrm{~cm}^{-1} \mathrm{~Hz}-1 / 2$ was achieved for ammonia in nitrogen at standard temperature and pressure, which corresponds to a detection limit of $0.25 \mathrm{ppbv}$.

\subsection{Photoacoustic spectroscopy}

The photoacoustic effect was discovered in 1880 by Alexander Graham Bell, who showed that thin discs emit sound when exposed to a beam of sunlight that was quickly interrupted with a rotating slotted disc. The absorbed energy from the light was transformed into kinetic energy in the sample and so a local heating and a pressure wave of sound arose. Later, Bell demonstrated the fact that materials exposed to different regions of the solar spectrum, that is, infrared and ultraviolet, can produce sounds too. Hence, by measuring the sound at different wavelengths, a photoacoustic spectrum from a sample can be recorded, so that it can be used to identify the absorbing components of the sample. This technique can be used to study solids, liquids and gases.

One of the uses of photoacoustic spectroscopy is the study of gas concentrations at the parts per billion or even parts per trillion levels. Although most photoacoustic detectors do not differ much from the original Bell's set-up, some enhancements have been incorporated in order to increase the sensitivity, such as enclosing the gaseous sample in a cylindrical chamber, and amplifying the sound signal by tuning the modulation frequency to an acoustic resonance of 
the sample cell, by using high-sensitive microphones together with lock-in techniques and utilizing intense lasers instead of sunlight to illuminate the sample.

Paldus et al. [44] reported photoacoustic spectroscopy on $\mathrm{NH}_{3}$ and $\mathrm{H}_{2} \mathrm{O}$ diluted in $\mathrm{N}_{2}$ using a CW DFB-QCL emitting at a wavelength of $8.5 \mu \mathrm{m}$. The noise-limited minimum detectable concentration of $\mathrm{NH}_{3}$ was 100 ppbv for an integration time of $1 \mathrm{~s}$. Lastly, Nägele et al. [45] built a multicomponent (ethane, methanol and ethanol) trace-gas monitoring system using QCLs as pump sources and a multipass photoacoustic cell.

\subsection{Other spectroscopies}

Other types of spectroscopies have also been used in the context of QCLs detection.

First, lamb-dip spectroscopy is a useful technique to study the spectra from polyatomic molecules. When a monochromatic light with a given wavelength passes through a chemical cell in a set-up similar to that depicted in Figure 6, a Gaussian absorption spectrum centred in the wavelength comes up. If we measure the absorption of a light beam (probe beam) by passing through two beams (pump and probe beams) of the same wavelength from opposite directions, by using a beam splitter, a less intense absorption is observed at that wavelength. This reduction in intensity appears as a dip, the so-called lamb-dip, and its position gives the location of the transition wavelength having no Doppler shift (Doppler-free in the absorption curve). Samman et al. [46] used a CW-operated DFB-QCL emitting at around $5.2 \mathrm{~mm}$ to obtain sub-Doppler resolution-limited saturation features in a lamb-dip experiment on NO. These lamb dips appeared as transition spikes with full-width at half maximum (FWHM) values around $4.3 \mathrm{MHz}$.

Gittins et al. [47] used a multimode Fabry-Perot-type QCL emitting at around $8.0 \mu \mathrm{m}$ wavelength for quantitative backscatter absorption measurements on isopropanol vapour. They developed and employed a pseudorandom code modulation of the laser, and explored its use for different absorption LIDAR (laser infrared detection and ranging).

Finally, Sonnenfroh et al. [48] used DFB-QCLs (1 around $5.4 \mu \mathrm{m})$ in quasi-CW mode close to room temperature in conjunction with a balanced radiometric detection technique to achieve high sensitivity, whereas Hvozdara et al. [49] demonstrated the first use of a GaAs-based QCL for gas-sensing applications.

\subsection{Direct detection applications: drug, explosive, plasma species and aerospace and military}

Drug detection is another important application field of QCL-based detection systems [50]. Lu et al. [51] demonstrated, by integrating an optoelectronic terahertz microsource into a glasssubstrated microchip within the near-field distance, a compact, label-free and non-invasive microbiosensing terahertz device that allowed detection of illicit drug powders with weight in the order of the nanogram.

The detection of explosive fingerprints is of great importance for security reasons. The detection of such substances has a number of drawbacks as explosive molecules are heavy, 
complex and have thousands of rotational and vibrational transitions. Furthermore, broadband features with no separation observed at low pressures happen in measured transmission spectra. Different explosives have, therefore, unique terahertz spectral fingerprints. Williams et al. [52] developed a high-power QCL emitting at around 4.4 THz. These lasers are based on a resonant-phonon depopulation scheme, and use a semi-insulating surface-plasmon waveguide. They managed a maximum power of $\sim 248$ and $138 \mathrm{~mW}$ pulsed and CW, respectively. van Neste et al. [53] have used two QCLs operating simultaneously with tunable wavelength windows matching the absorption peaks of analytes in order to improve sensitivities of standoff spectroscopy techniques, leading to a sensitivity of $100 \mathrm{ng} / \mathrm{cm}^{2}$ and a standoff detection distance of $20 \mathrm{~m}$ for surface-adsorbed analytes such as explosives and tributyl phosphate.

Molecular plasmas are tools in plasma-enhanced chemical vapour deposition and in etching systems to deposit or remove thin films. The analysis of the precursor gas fragmentation and the monitoring of plasma reaction products is the key to an improved understanding and control of these chemical-active discharges. This observation can be done by using absorption spectroscopy methods in the mid-infrared spectral region. Recently, a QCL-based absorption spectroscopic system, the quantum cascade laser measurement and control system (Q-MACS), was developed and used to study industrial process plasmas and for environmental studies, and its applicability for monitoring online processes has been proven [54].

Finally, QCLs are also applied to aerospace and military uses. For instance, the first of a new generation of miniature tunable laser mid-IR spectrometers operating at room temperature for in situ measurement of atmospheric and evolved planetary gases started to develop not too long ago. These devices are based on newly available room-temperature QCL sources in the 3-12 $\mu \mathrm{m}$ wavelength region and have immediate applications to Mars, Titan, Venus and Europa missions, being operated on a descending or penetrating probe, lander, rover or aerobot, and would consume only a few watts of power, with a weight less than $1 \mathrm{~kg}$ [55]. Furthermore, features of QCL make them good laser sources to carry out non-destructive-imaging engine combustors, where failure mechanisms, engine performance in aircraft and moisture content in jet fuel are examined. A low thermal noise background is required for such purpose, and ceramic ports exist already on such platforms transparent to terahertz radiation. Scale model radar cross-section experiments have also been carried out in the aerospace industry, to replace the bulky and extremely inefficient molecular lasers with QCLs, at the University of Massachusetts Lowell Sub-Millimetre Wave Laboratory [56], to perform scale model radar crosssection measurements for phenomenology and target-recognition database formation.

The infrared spectrum is also used in the aerospace industry for detection purposes, for instance, in the infrared scene generation, which is presently a critical technology for testing of infrared-imaging systems, for example, in infrared-guided missile systems, and QCLs could have an application in this technology to replace large- and slow-response 'resistor banks' with tailored design QCLs, to mimic the thermal background of a given scene [57]. Moreover, coherent transceiver using a terahertz quantum cascade laser (TQCL) as the transmitter and an optically pumped molecular laser as the local oscillator has been used for imaging purposes along with inverse synthetic aperture radar (ISAR), in which the range of the target was limited 
by the TQCL power (around 10-4 W) and indoor atmospheric attenuation at $2.408 \mathrm{THz}$, leading to a coherence length of the transmitter of up to several hundred metres [58].

\section{State of the art and outlook}

QCLs are also currently facing a number of challenges, which will be summarized below.

First, the extensions of the wavelength range into the far-infrared. Rochat et al. [59] grew farinfrared QC structures based on a vertical transition active region emitting at 1 around $76 \mu \mathrm{m}$. This is a challenging issue, so long as population inversion is difficult to attain for such long wavelengths. Energy levels are generally so close that selective injection into a single level is difficult. Furthermore, LO-phonon scattering is replaced by electron-electron scattering as the dominant electron relaxation mechanism, which is more difficult to model accurately. Moreover, wavewide losses are expected to be high, although even very low-temperature QCL operation would be a great accomplishment due to the lack of narrow band and high-power compact sources in the far-infrared wavelength range. In fact, temperature is a key player when it is required to achieve a certain emission wavelength as Figure 7 shows.

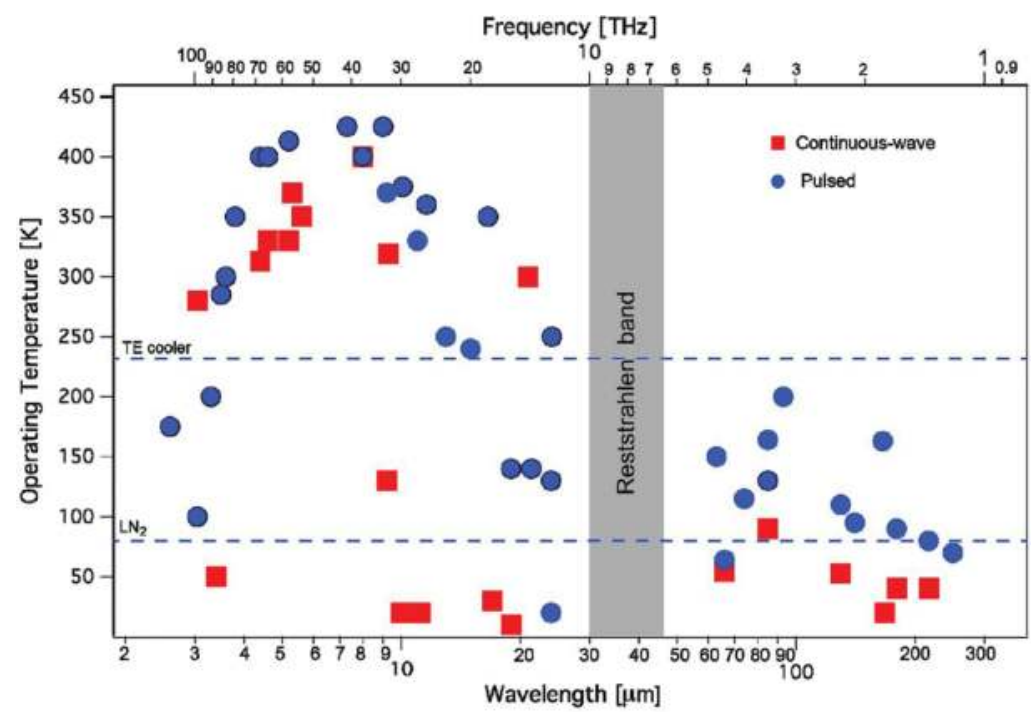

Figure 7. Operating temperature plot as a function of the emission wavelength/frequency for QCLs [60].

Another challenge would also be to fibre-optic wavelengths. In fact, the fabrication of large optical waveguides, powers of 14 and $5 \mathrm{~W}$ at respective temperatures of 15 and $280 \mathrm{~K}$, is demonstrated at a wavelength of approximately $5.2 \mu \mathrm{m}$ [61].

On the other side of the IR spectrum, reaching shorter wavelengths is another motivation for a more selection of materials. Work on this respect has also be done on GaN-based devices [61], 
with Gmachl et al. [62] having measured intersubband optical absorption in narrow (15-30 wide), GaN/AlGaN quantum wells grown by MBE on sapphire substrate. Peak absorption wavelengths ranged from $4.2 \mu \mathrm{m}$ for $30 \AA$ wide wells to $1.77 \mu \mathrm{m}$ for a $15 \AA$ wide well. On the other hand, lizuka et al. [63] reported ultrafast intersubband relaxation (less than $150 \mathrm{fs}$ ) at a wavelength of $4.5 \mu \mathrm{m}$ in $\mathrm{Al}_{0.65} \mathrm{Ga}_{0.35} \mathrm{~N} / \mathrm{GaN}$ MQWs, with as many as $200 \mathrm{QWs}$, making this result promising for fabrication ultrafast optical switches.

Doping level of the active region is a key optimization parameter that determines maximum drive current, optical losses and threshold current. Faist et al. [61] presented a systematic change of the active region doping in an InAlAs-InGaAs/InP lasers emitting at $9 \mu \mathrm{m}$. On the other hand, the wavelength tunability of each QC-DFB laser is especially limited to cover the entire molecular absorption spectrum of volatile organic compounds and hydrocarbons, which could be addressed by separating the gain medium from the wavelength-selective element [61].

Other challenges involve the low conversion efficiencies $(<1 \%)$ between electrical and optical power, also known as wall plug efficiency (WPE) [64], and also the unavoidable fact that farinfrared (terahertz) QCLs lack proper performance at room-temperature operation. Belkin et al. [65] have recently reviewed recent research that has led to a new class of QCL light sources that has overcome these limitations leading to room-temperature operation in the terahertz spectral range, with nearly $2 \mathrm{~mW}$ of optical power and significant tunability by using intracavity $\mathrm{THz}$ difference-frequency generation (DFG) in dual-wavelength mid-IR QCLs. However, Lu et al. [66] recently presented a strong-coupled strain-balanced quantum cascade laser design for efficient $\mathrm{THz}$ generation based on intra-cavity difference-frequency generation, demonstrating continuous-wave, single-mode $\mathrm{THz}$ emissions with a wide-frequency tuning range of $2.06-4.35 \mathrm{THz}$ and an output power up to $4.2 \mu \mathrm{W}$ at room temperature from two monolithic three-section sampled grating distributed feedback-distributed Bragg reflector lasers.

Furthermore, Burghoff et al. [67] demonstrated frequency combs based on terahertz QCLs, combining high power of lasers with the broadband capabilities of pulsed sources. By fully exploiting the quantum-mechanically broadened gain spectrum available to these lasers, $5 \mathrm{~mW}$ of terahertz power spread across 70 laser lines can be generated. Therefore, the radiation is sufficiently powerful to be detected by Schottky-diode mixers, and will lead to compact terahertz spectrometers.

About gas detection, Harrer et al. [68] combined the operation mode and low-divergence emission from QCLs with two-dimensional array integration with multiple emission and detection frequencies leading to detecting propane concentrations of $0-70$ and $0-90 \%$ for isobutane at a laser operation wavelength of $6.5 \mu \mathrm{m}$ using a $10 \mathrm{~cm}$ gas cell in double-pass configuration.

Finally, possibly the best way to assess the current state of the art for volume production of QCLs is to start from the market requirement from the end-user point of view. An example is the continuous emission monitoring (CEM) market, which includes engine emission and power-plant stack monitoring, is fiercely competitive, with a range of different gas-sensing technologies, such as Fourier transform spectrometry, chemiluminescence, non-dispersive 
infrared, and so on, each trying to increase its market share. Among these technologies, QCLbased gas sensor technology is still in its infancy, since although predictions indicate a great improvement in performance, much demonstration and convincing is still required in this conservative market. Moreover, it is difficult to justify a system whose price is above the market, despite better performance, due to the risk of an unproven and new technology. Hence, a QCL device might be priced below the market, which can have negative consequences that can reach the component supplier. Customers are usually reluctant to acquire a new sensor having other well-proven systems already in the market. Therefore, demonstration of the unique advantages of the QCL-based devices is often necessary, including specifications, performance, mean time to failure (MTTF) parameters, traceability and warranty period with a significant impact [69]. Implementation of high-volume production and proper validation processes on QCLs are producing positive outcomes. Along with a noticeable improvement in gas-sensing performance parameters, volume-cost reduction is allowing QCL systems price decrease to meet market demands. By consistently tackling the challenges of the QCL volume manufacturing, carrying out severe testing procedures, implementing quality-control systems and reaching adequate device costing, QCL developers and manufacturers can complete and come into the marketplace.

Finally, continuing to build consumer confidence in QCL commercial products, it should be easier for customers to digest the unavoidable contract 'warranty' and 'product liability' clauses found in supply contracts [69].

\section{Summary}

In this chapter, an overview of quantum cascade lasers (QCLs) is presented. A historical introduction is first introduced. The basic features of QCLs are outlined, as well as a brief description of the issues that this work deals with. The operation and fundamentals of QCLs are also described. An analysis of the operation of these structures is included. Basically, the use of superlattices and tunability due to the layer thickness are the key features, in conjunction with the intersubband tunnelling transition - cascade. The QCLs are usually three-level lasers. Rate equations are also included. Furthermore, introducing a graphical brief timeline (Figure 8), including some of the most important milestones achieved in the world of QCL, would be helpful in this section.

An overview over the materials used for these devices is also included, that is, InGaAs and InAlAs layers, InAs/AlSb, Si/SiGe and GaN-based.

QCLs have a wide range of applications: first, trace-gas detection by optical methods in the mid-infrared, the great suitability of the TILDAS techniques and DFB-QCLs in trace-gassensing applications. 


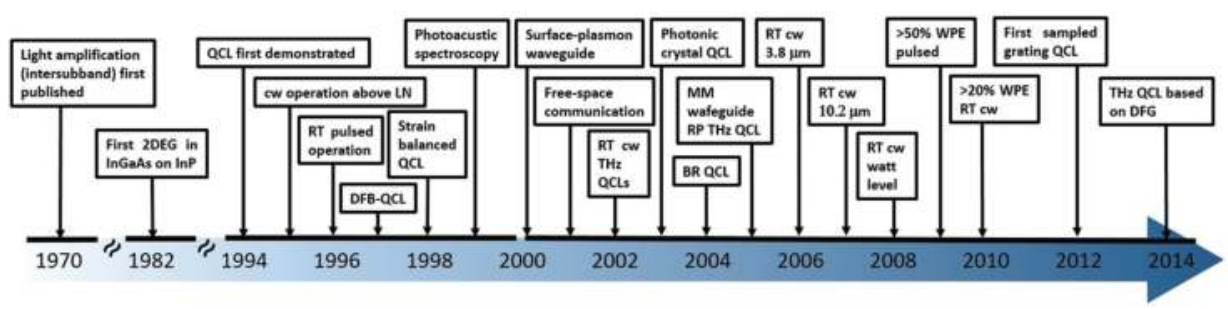

Figure 8. Achievements of QCLs over time.

Cavity ring-down spectroscopy is another technique used for gas detection and briefly described above. QCLs are also applied in photoacoustic spectroscopy, to study gas concentrations at the parts per billion or even parts per trillion levels. Applications in gas detection using QCLs with other types of spectroscopy and variations, such as lamb-dip spectroscopy, radiometric detection techniques, and so on, are mentioned.

The latest advances in QCL applications are also described. The extension of the wavelength range into the far-infrared but also shorter wavelengths, fibre-optic wavelengths and fabrication of large optical waveguides, doping issues, room-temperature operation problems, frequency combs based on terahertz devices, new insights on gas detection and finally current state of the art for volume production of QCLs are mentioned.

\section{Author details}

Raúl Pecharromán-Gallego*

Address all correspondence to: anubis_rpg@hotmail.com

The Last Push Consulting, Heverlee, Leuven, Belgium

\section{References}

[1] Esaki L, Tsu R. Superlattice and negative differential conductivity in semiconductors. IBM Journal of Research and Development. 1970;14(1):61-65. DOI: 10.1147/rd.141.0061.

[2] Kazarinov R, Suris R. Possibility of the amplification of electromagnetic waves in a semiconductor with a superlattice. Soviet Physics - Semiconductors. 1971;5(4):707-709.

[3] Faist J, Capasso F, Sivco D L, Sirtori C, Hutchinson A L, Cho A Y. Quantum cascade laser. Science. 1994;264:553-556. DOI: 10.1126/science.264.5158.553. 
[4] Markus-Christian Amann. Semiconductor Technology (E26) [Internet]. [Updated: 2016]. Available from: https://www.wsi.tum.de/Research/AmanngroupE26/AreasofResearch/tabid/115/Default.aspx [Accessed: 12-05-2016].

[5] Capasso F, Gmachl C, Sivco D L, Cho Q Y. Quantum cascade lasers. Physics World. 1999;12(6):27-33. DOI: http://dx.doi.org/10.1088/2058-7058/12/6/26.

[6] Alpes Lasers SA. Alpes Lasers-Quantum Cascade Lasers [Internet]. [Updated: 2016]. Available from: http://www.alpeslasers.ch/ [Accessed: 12-05-2016].

[7] Tredicucci A, Gmachl C, Capasso F, Sivco D L, Hutchinson A L, Cho A Y. A multiwavelength semiconductor laser. Nature. 1998;396:350-353. DOI: 10.1038/24585.

[8] Kosterev A A, Tittel F K, Köhler R, Gmachl C, Capasso F, Sivco D L, Cho A Y, Wehe S, Allen M G. Thermoelectrically cooled quantum-cascade-laser-based sensor for the continuous monitoring of ambient atmospheric carbon monoxide. Applied Optics. 2002;41(6):1169-1173. DOI: 10.1364/AO.41.001169.

[9] Edgar J H, Strite S, Akasaki I, Amano H, Wetzel C, editors. Properties processing and applications of gallium nitride and related semiconductors. 1st ed. UK: EMIS datareviews series no 23. INSPEC; 1999. 830 p. DOI: ISBN-10: 0852969538. ISBN-13: 978-0852969533.

[10] Beernik K J, Thornton R L, Chung H F. Low threshold current dual wavelength planar buried heterostructure lasers with close spatial and large spectral separation. Applied Physics Letters. 1994;64(9):1082-1084. DOI: 10.1063/1.110939.

[11] Dutta N K, Cella T, Zilko J L, Ackerman D A, Piccirilli A B, Greene L I. InGaAsP closely spaced dual wavelength laser. Applied Physics Letters. 1986;48(25):1725-1726. DOI: $10.1063 / 1.96815$.

[12] Pellandini P, Stanley R P, Houdre R, Oesterle U, Ilegems M, Weisbuch C. Dualwavelength laser emission from a coupled semiconductor microcavity. Applied Physics Letters. 1997;71(7):864-866. DOI: 10.1063/1.119671.

[13] Gmachl C, Sivco D L, Baillargeon J N, Hutchinson A L, Capasso F, Cho A Y. Quantum cascade lasers with a heterogeneous cascade: two-wavelength operation. Applied Physics Letters. 2001;79(5):572-574. DOI: 10.1063/1.1383806.

[14] \#Köhler R, Gmachl C, Tredicucci A, Capasso F, Sivco D L, Chu S N G, Cho A Y. Singlemode tunable, pulsed, and continuous wave quantum-cascade distributed feedback lasers at $\lambda \cong 4.6-4.7 \mu \mathrm{m}$. Applied Physics Letters. 2000;79(9):1092-1094. DOI: 10.1063/1.125987.

[15] Banerjee S, Spencer P S, Shore K A. Design of a tunable quantum cascade laser with enhanced optical non-linearities. IEE Proceedings Optoelectronics. 2006;153(1):40-42. DOI: 10.1049/ip-opt:20050061.

[16] Gmachl C, Capasso F, Sivco D L, Cho A Y. Recent progress in quantum cascade lasers and applications. Report on Progress in Physics. 2001;64(11):1533-1601. DOI: $10.1088 / 0034-4885 / 64 / 11 / 204$. 
[17] Walther C, Scalari G, Faist J, Beere H, Ritchie D. Low frequency terahertz quantum cascade laser operating from 1.6 to 1.8THz. Applied Physics Letters. 2006;89(23): 231121-3. DOI: 10.1063/1.2404598.

[18] Revin D G, Cockburn J W, Steer M J, Airey R J, Hopkinson M, Krysa A B, Wilson R, Menzel S. InGaAs/AlAsSb/InP quantum cascade lasers operating at wavelengths close to 3 m. Applied Physics Letters. 2007;90(2):021108-3. DOI: 10.1063/1.2431035.

[19] Green R, Roberts J, Krysa A, Wilson L, Cockburn J, Revin D, Zibik E, Carder D, Airey P. MOVPE grown quantum cascade lasers. Physica E: Low-dimensional Systems and Nanostructures. 2004;21(2-4):863-866. DOI: 10.1016/j.physe.2003.11.133.

[20] Liu Z., Wasserman D, Howard S S, Hoffman A J, Gmachl C F, Wang X, Tanbun-Ek T, Cheng L, Choa F-S. Room-temperature continuous-wave quantum cascade lasers grown by MOCVD without lateral regrowth. IEEE Photonics Technology Letters. 2006;18(12):1347-1349. DOI: 10.1109/LPT.2006.877006.

[21] Diehl L, Bour D, Corzine S, Zhu J, Höfler G, Lončar M, Troccoli M, Capasso F. Hightemperature continuous wave operation of strain-balanced quantum cascade lasers grown by metal organic vapor-phase epitaxy. Applied Physics Letters. 2006;89(8): 081101-3. DOI: 10.1063/1.2337284.

[22] Teissier R, Barate D, Vicet A, Alibert C, Baranov B A, Marcadet X, Renard X, Garcia M, Sirtori C, Revin D, Cockburn J. Room temperature operation of InAs/AlSb. Applied Physics Letter. 2004;85(2):167-169. DOI: 10.1063/1.1768306.

[23] Ohtani K, Fujita K, Ohno H. Room-temperature InAs/AlSb quantum-cascade laser operating at $8.9 \mu \mathrm{m}$. Electronics Letters. 2007;43(9):520-522. DOI: 10.1049/el:20070251.

[24] Lynch S A, Bates R, Paul D J, Norris D J, Cullis A G, Ikonic Z, Kelsall R W, Harrison P, Arnone D D, Pidgeon C R. Intersubband electroluminescence from Si/SiGe cascade emitters at terahertz frequencies. Applied Physics Letters. 2002;81(9):1543-1545. DOI: 10.1063/1.1501759.

[25] De Rossi A, Carras M, Paul D J. Low-loss surface-mode waveguides for terahertz SiSiGe quantum cascade lasers. IEEE Journal of Quantum Electronics. 2006;42(2):12331238. DOI: 10.1109/JQE.2006.883496.

[26] Paul D J. Si/SiGe heterostructures: from material and physics to devices and circuits. Semiconductor Science and Technology. 2004;19(10):75-108. DOI: 10.1088/0268-1242/ 19/10/R02.

[27] Pecharromán-Gallego R. Investigations of the luminescence of $\mathrm{GaN}$ and InGaN/GaN quantum wells [thesis]. UK: University of Strathclyde; 2004. 216 p. Available from: http://suprimo.lib.strath.ac.uk/primo_library/libweb/action/display.do?tabs=detailsTab\&ct=display\&fn=search\&doc=SUVOY682303\&ind $x=1 \&$ recIds=SUVOY682303\&recIdxs=0\&elementId=0\&renderMode=poppedOut\&displayMode=full \&frbrVersion=\&dscnt=1\&scp.scps=scope $\% 3 \mathrm{~A} \% 28$ SU $\% 29 \% 2$ Cscope $\% 3 \mathrm{~A} \% 28 \mathrm{cla}$ $\% 29 \&$ frbg $=\&$ tab $=$ local \&dstmp $=1463141375653 \&$ srt $=$ rank\&mode $=$ Ba- 
sic\&dum $=$ true\&tb=t\&vl(freeText0)=Pecharroman\&vid=SUVU01 DOI: Thesis no : T10902.

[28] Sun G, Soref R A, Khurgin J B. Active region design of a terahertz GaN/Al0.15Ga0.85N quantum cascade laser. Superlattices and Microstructures. 2005;37(2):107-113. DOI: 10.1016/j.spmi.2004.09.046.

[29] Heber J D, Gmachl C, Ng H M, Cho A Y. Comparative study of ultrafast intersubband electron scattering times at $\sim 1.55 \mu \mathrm{m}$. Applied Physics Letters. 2002;81(7):1237-1239. DOI: $10.1063 / 1.1500412$.

[30] Huang G S, Lu T C, Yao H H, Kuo H C, Wang S C, Sun G, Lin C-W, Chang L, Soref R A. GaN/AlGaN active regions for terahertz quantum cascade lasers grown by lowpressure metal organic vapor deposition. Journal of Crystal Growth. 2007;298(1):687390. DOI: 10.1016/j.jcrysgro.2006.10.106.

[31] Wang S C, Soref R, Sun G. Fabrication and characteristics of GaN/AlGaN multilayer structure for terahertz quantum-cascade laser. SPIE Proceedings. Terahertz Physics, Devices, and Systems. 2006;6373:637309. DOI: 10.1117/12.685678.

[32] Lopez-Otero A. Hot wall epitaxy. Thin Solid Films. 1978;49(1):3-57. DOI: 10.1016/0040-6090(78)90309-7.

[33] Inoue Y, Nagasawa H, Sone N, Ishino K, Ishida A, Fujiyasu H, Kim J J, Makino H, Yao T, Sakakibara S, Kuwabara M. Fabrication and characterization of short period AlN/GaN quantum cascade laser structures. Journal of Crystal Growth. 2004;265(1-2): 65-70. DOI: 10.1016/j.jcrysgro.2004.01.044.

[34] Ishida A, Ose T, Nagasawa H, Ishino K, Inoue Y, Fujiyasu H. Quantum-cascade structure in AlN/GaN system assisted by piezo-electric effect. Japanese Journal of Applied Physics. 2002;41(2, 3A):236-238. DOI: 10.1143/JJAP.41.L236.

[35] Ishida A, Ose T, Nagasawa H, Inoue Y, Tatsuoka H, Fujiyasu H, Ko H-J, Makino H, Yao T, Kan H. Characterization of AlN/GaN quantum-cascade structures prepared by hotwall epitaxy. Physica Status Solidi C: Current Topics in Solid State Physics. 2002;0(1): 520-523. DOI: 10.1002/pssc.200390103.

[36] Sigrist M W, editor. Air monitoring by spectroscopic techniques. United States: New York, NY: Wiley-Interscience; 1994. 531 p. DOI: ISBN 0471558753, 9780471558750.

[37] Namjou K, Cai S, Whittaker E A, Faist J, Gmachl C, Capasso F, Sivco D L, Cho A Y. Sensitive absorption spectroscopy with a room-temperature distributed-feedback quantum-cascade laser. Optics Letters. 1998;23(3):219-221. DOI: 10.1364/OL.23.000219.

[38] Faist J, Gmachl C, Capasso F, Sirtori C, Sivco D L, Billargeon J N, Cho A Y. Distributed feedback quantum cascade lasers. Applied Physics Letters. 1997;70(20):2670-2673. DOI: 10.1063/1.119208.

[39] Sharpe S W, Kelly J F, Hartman J S, Gmachl C, Capasso F, Sivco D L, Baillargeon J N, Cho A Y. High-resolution (Doppler-limited) spectroscopy using quantum-cascade 
distributed-feedback lasers. Optics Letters. 1998;23(17):1396-1398. DOI: 10.1364/OL. 23.001396.

[40] Williams R M, Kelly J F, Hartman J S, Sharpe S W, Taubman M S, Hall J L, Capasso F, Gmachl C, Sivco D L, Baillargeon J N, Cho A Y. Kilohertz linewidth from frequencystabilized mid-infrared quantum cascade lasers. Optics Letters. 1999;24(24):1844-1866. DOI: 10.1364/OL.24.001844.

[41] Kosterev A A, Curl R F, Tittel F K, Gmachl C, Capasso F, Sivco D, Baillargeon J N, Hutchinson A L, Cho A Y. Effective utilization of quantum-cascade distributedfeedback lasers in absorption spectroscopy. Applied Optics. 2000;39(24):4425-4430. DOI: 10.1364/AO.39.004425.

[42] Kosterev A A, Curl R F, Tittel F K, Gmachl C, Capasso F, Sivco D L, Baillargeon J N, Hutchinson A L, Cho A Y. Absorption spectroscopy with quantum cascade lasers. Laser Physics. 2001;11(1):39-49. DOI: PMID 12143896.

[43] Paldus B A, Harb C C, Spence T G, Zare R N, Gmachl C, Capasso F, Sivco D L, BaillargeonJ N, Hutchinson A L, Cho A Y. Cavity ringdown spectroscopy using mid-infrared quantum-cascade lasers. Optics Letters. 2000;25(9):666-668. DOI: 10.1364/OL. 25.000666 .

[44] Paldus B A, Spence T G, Zare R N, Oomens J, Harren F J M, Parker D H, Gmachl C, Cappasso F, Sivco D L, Baillargeon J N, Hutchinson A L, Cho A Y. Photoacoustic spectroscopy using quantum-cascade lasers. Optics Letters. 1999;24(3):178-180. DOI: 10.1364/OL.24.000178.

[45] Nägele M, Hofstetter D, Faist J, Sigrist M W. Mobile laser photoacoustic spectrometer for multicomponent trace-gas monitoring based on $\mathrm{CO}_{2}$ - and quantum-cascade lasers as pump sources. In: Conference on Lasers and Electro-Optics Europe; 10-15 September, 2000; Nice, France. IEEE; 2000. p. 12-22. DOI: 10.1109/CLEOE.2000.909779.

[46] Samman A, Rimai L, McBride J R, Carter R O, Weber W H, Gmachl C, Capasso F, Hutchinson A L, Sivco D L, Cho A Y. Potential use of near, mid and far infrared laser diodes in automotive LIDAR applications. In: 52nd Vehicular Technology Conference; 24-28 September, 2000; Boston. USA. IEEE; 2000. p. 2084-2089, vol. 5. DOI: 10.1109/ VETECF.2000.883239.

[47] Gittins C M, Wetjen E T, Gmachl C, Capasso F, Hutchinson A L, Sivco D L, Baillargeon J N, Cho A Y. Quantitative gas sensing by backscatter-absorption measurements of a pseudorandom code modulated $\lambda \sim 8$ - $\mu \mathrm{m}$ quantum cascade laser. Optics Letters. 2000;25(16):1162-1164. DOI: 10.1364/OL.25.001162.

[48] Sonnenfroh D M, Rawlins W T, Allen M G, Gmachl C, Capasso F, Hutchinson A L, Sivco D L, Baillargeon J N, Cho A Y. Application of balanced detection to absorption measurements of trace gases with room-temperature, quasi-CW quantum-cascade lasers. Applied Optics. 2001;40(6):812-820. DOI: 10.1364/AO.40.000812. 
[49] Hvozdara L, Gianordoli S, Strasser G, Schrenk W, Unterrainer K, Gornik E, Murthy C S S S, Kraft M, Pustogow V, Mizaikoff B, Inberg A, Croitoru N. Spectroscopy in the gas phase with GaAs/AlGaAs quantum-cascade lasers. Applied Optics. 2000;39(36):69266930. DOI: 10.1364/AO.39.006926.

[50] Kawase K. Terahertz imaging for drug detection and large-scale integrated circuit inspection. Optics and Photonics News. 2004;15(10):34-39. DOI: 10.1364/OPN. 15.10.000034.

[51] Lu J Y, Chen L J, Kao T F, Chang H H, Chen H W, Liu A S, Chen Y C, Wu R B, Liu W S, Chyi J I, Sun C K. Terahertz microchip for illicit drug detection. IEEE Photonics Technology Letters. 2006;18(21):2254-2256. DOI: 10.1109/LPT.2006.883285.

[52] Williams B S, Kumar S, Hu Q, Reno J L. High-power terahertz quantum-cascade lasers. Electronics Letters. 2006;42(2):89-91. DOI: 10.1049/el:20063921.

[53] van Neste C W, Senesac L R, Thundat T. Standoff spectroscopy of surface adsorbed chemicals. Analytical Chemistry. 2009;81(5):1952-1956. DOI: 10.1021/ac802364e.

[54] Röpcke J, Davies P B, Glitsch S, Hempel F, Macherius U, Lang N, Rousseau A, Stancu GD, Weltmann K-D, Welzel S, Zimmermann H. Q-MACS, A compact quantum cascade laser absorption spectroscopy system for plasma diagnostics. In: The 2nd International Workshop on Quantum Cascade Lasers; 6-9 September 2006; Brindisi, Italy. Massachusetts Institute of Technology; 2006. p. 388-395.

[55] Jet Propulsion Laboratory. Mars Science Laboratory [Internet]. Available from: http:// mars.jpl.nasa.gov/msl/ [Accessed: 19-05-2016].

[56] University of Massachusetts Lowell Sub-Millimetre Wave Laboratory. Sub-Millimetre Wave Laboratory I UMass Lowell [Internet]. Available from: https://www.uml.edu/ Research/STL/ [Accessed: 19-05-2016].

[57] Nelson T R. USAF applications for quantum cascade lasers. In: The 2nd International Workshop on Quantum Cascade Lasers; 6-9 September 2006; Brindisi, Italy. Massachusetts Institute of Technology; 2006. p. 455-465.

[58] Danylov A A, Goyette T M, Waldman J, Coulombe M J, Gatesman A J, Giles R H, Qian X, Chandrayan N, Vangala S, Termkoa K, Goodhue W D, Nixon W E. Terahertz inverse synthetic aperture radar (ISAR) imaging with a quantum cascade laser transmitter. Optics Express. 2010;18(15):16264-16272. DOI: 10.1364/OE.18.016264.

[59] Rochat M, Beck M, Faist J, Oesterle U. Measurement of far-infrared waveguide loss using a multisection single-pass technique. Applied Physics Letters. 2001;78(14):19671969. DOI: $10.1063 / 1.1357444$.

[60] Vitiello M S, Scalari G, Williams B, Paolo D-N. Quantum cascade lasers: 20 years of challenges. Optics Express. 2015;23(4):5167-5182. DOI: 10.1364/OE.23.005167. 
[61] Faist J, Aellen T, Gresch T, Beck M, Giovannini M. Mid-infrared coherent sources and applications. NATO Science for Peace and Security Series ed. The Netherlands: Springer; 2008. 630 p. DOI: ISBN: 978-1-4020-6463-0.

[62] Gmachl C, Ng H M, Cho A Y. Intersubband absorption in GaN/AlGaN multiple quantum wells in the wavelength range of $\lambda \sim 1.75-4.2 \mu \mathrm{m}$. Applied Physics Letters. 2000;77(3):334-336. DOI: 10.1063/1.126968.

[63] Iizuka N, Kaneko K, Suzuki N, Asano T, Noda S, Wada O. Ultrafast intersubband relaxation ( $\leq 150 \mathrm{fs}$ ) in AlGaN/GaN multiple quantum wells. Applied Physics Letters. 2000;77(5):648-650. DOI: 10.1063/1.127073.

[64] Yao A Y, Hoffman J, Gmachl C. Mid-infrared quantum cascade lasers. Nature Photonics. 2012;6(7):432-439. DOI: 10.1038/nphoton.2012.143.

[65] Belkin M A, Capasso F. New frontiers in quantum cascade lasers: high performance room temperature terahertz sources. Physica Scripta. 2015;90(11):118002. DOI: 10.1088/0031-8949/90/11/118002.

[66] Lu Q, Wu D, Sengupta, Slivken S, Razeghi M. Room temperature continuous wave, monolithic tunable $\mathrm{THz}$ sources based on highly efficient mid-infrared quantum cascade lasers. Scientific Reports. 2016;6:23595. DOI: 10.1038/srep23595.

[67] Burghoff D, Kao T-Y, Han N, Chan C W I, Cai X, Yang Y, Hayton D J, Gao J-R, Reno J L, Hu Q. Terahertz laser frequency combs. Nature Photonics. 2014;8:462-467. DOI: 10.1038/nphoton.2014.85.

[68] Harrer A, Szedlak R, Schwarz B, Moser H, Zederbauer T, MacFarland D, Detz H, Andrews A M, Schrenk W, Lendl B. Mid-infrared surface transmitting and detecting quantum cascade device for gas-sensing. Scientific Reports. 2016;6:21795. DOI: 10.1038/ srep21795.

[69] Normand E, Howieson I. Quantum-cascade lasers enable gas-sensing technology. Laser Focus World. 2007;43(4):90-92. 